\title{
Saúde mental de cuidadores de crianças e adolescentes com neoplasia maligna no Nordeste do Brasil
}

\author{
Mental health of caregivers from children and teenagers with maligna neoplasia in \\ Northeast Brazil
}
Salud mental de cuidadores de niños y adolescentes con neoplasia maligna en el Noreste de Brasil

Walter Marcelo Oliveira de Carvalho ${ }^{1 * *}$, Rute Nascimento da Silva1, Yasmin Oliveira Santos ${ }^{1}$, Manuela Naiane Lima Barreto ${ }^{1}$, Louise Náder Santos Silva ${ }^{1}$, Marcos Vinícius Ribeiro Nascimento ${ }^{1}$, Renata Isabela Feitosa de Carvalho Nascimento ${ }^{1}$, Marcela Beatriz Feitosa de Carvalho ${ }^{1}$, Ana Jovina Barreto Bispo ${ }^{1 *}$, Carla Viviane Freitas de Jesus ${ }^{1}$, Verónica de Lourdes Sierpe Jeraldo ${ }^{1}$.

\section{RESUMO}

Objetivo: Avaliar características sociodemográficas e os sintomas de ansiedade/depressão em cuidadores de crianças e adolescentes com neoplasias malignas. Métodos: Estudo prospectivo, com cuidadores de pacientes de 0 a 19 anos de idade em tratamento oncológico, em um serviço de referência em saúde do câncer, em uma capital do nordeste brasileiro. O Inventário de Ansiedade/Depressão de Beck (BDI) foi aplicado para avaliar a presença e o nível de ansiedade/depressão do cuidador e o questionário Caregiver Burden Scale para analisar aspectos gerais da qualidade de vida do cuidador. Resultados: Participaram do estudo 45 cuidadores, com predominância da mãe como cuidadora $(91,1 \%)$, idade média de 34 anos e renda familiar abaixo de um salário-mínimo. A maioria apresentava sintomas graves de ansiedade e os sinais de depressão eram mínimos, apresentando Nível de Estresse Grau I com baixo risco de sintomas físicos e psicológicos. Não houve associação entre os níveis de ansiedade e a escala de sobrecarga do cuidador ( $p=$ $0,163)$. Por outro lado, há uma correlação entre depressão e sobrecarga do cuidador $(p<0,001)$. Conclusão: Observouse cuidadores com sinais e sintomas graves de ansiedade, de depressão leve, com baixo estresse e sem risco de sintomas físicos e psicossomáticos.

Palavras-chave: Câncer, Ansiedade, Depressão.

\section{ABSTRACT}

Objective: To evaluate sociodemographic characteristics and symptoms of anxiety/depression in caregivers of children and adolescents diagnosed with malignant neoplasms. Methods: Prospective study, by convenience sampling, with caregivers of patients from 0 to 19 years of age undergoing cancer treatment, in a reference service in cancer health, in a capital of northeastern Brazil. The Beck Anxiety/Depression Inventory (BDI) was applied to assess the presence and level of anxiety depression of the caregiver and the Caregiver Burden Scale questionnaire to analyze general aspects of the caregiver's quality of life. Results: 45 caregivers participated in the study, with a predominance of the mother as caregiver $(91.1 \%)$, mean age of 34 years and family income below the minimum wage. Most had severe symptoms of anxiety and signs of tendency according to the Grade I Stress level pattern with a low risk of physical and psychological symptoms. There was no association between anxiety levels and the caregiver's burden scale $(p=0.163)$. On the other hand, there is a correlation between depression and caregiver burden $(p<0.001)$. Conclusion: Caregivers were observed with severe signs and symptoms of anxiety, mild depression, with low stress and without risk of physical and psychosomatic symptoms.

Keywords: Cancer, Anxiety, Adjustment disorders.

\section{RESUMEN}

Objetivo: Evaluar características sociodemográficas y síntomas de ansiedad/depresión en cuidadores de niños y adolescentes diagnosticados de neoplasias malignas. Métodos: Estudio prospectivo, por muestreo de conveniencia, con cuidadores de pacientes de 0 a 19 años en tratamiento oncológico, en un servicio de referencia en salud oncológica, en una ciudad capital del noreste de Brasil. Se aplicó el Inventario de Ansiedad/Depresión de Beck (BDI) para evaluar la presencia y nivel de ansiedade/depresión del cuidador y el cuestionario Caregiver Burden Scale para analizar aspectos generales de la calidad de vida del cuidador. Resultados: En el estudio participaron 45 cuidadores, con predominio de la madre como cuidadora $(91,1 \%)$, edad media de 34 años e ingresos familiares por debajo del salario mínimo. La mayoría presentaba síntomas graves de ansiedad y los signos de depresión eran mínimos, con un Nivel de Estrés de Nivel I con un riesgo bajo de síntomas físicos y psicológicos. No hubo asociación entre los niveles de ansiedad y la escala de sobrecarga del cuidador $(\mathrm{p}=0,163)$. Por otro lado, existe una correlación entre la depresión y la sobrecarga del cuidador $(p<0,001)$. Conclusión: Se observó a los cuidadores con signos y síntomas severos de ansiedad, depresión leve, con poco estrés y sin riesgo de síntomas físicos y psicosomáticos.

Palabras clave: Cáncer, Ansiedad, Depresión.

\footnotetext{
${ }^{1}$ Universidade Tiradentes (UNIT), Aracaju - SE. *E-mail: anajovina70@gmail.com.

** In memoriam.
} 


\section{INTRODUÇÃO}

Extremamente raro quando comparado aos cânceres de adultos, o câncer juvenil (CJ) representa entre $2 \%$ e $3 \%$ de todos os tumores malignos no mundo. Todos os anos, 300.000 casos de CJ são diagnosticados em todo o mundo em menores de 19 anos. Os Estados Unidos da América (EUA) apresentaram uma incidência de 17,4/100.000 crianças e adolescentes a cada ano (NAM GE, et al., 2016; KARLSON CW, et al., 2015).

O CJ é uma doença crescente. Na Colômbia, a incidência aumentou entre 2002 e 2006, resultando em 70.877 novos casos no período (PALACIO C, et al., 2018). A Indonésia alcançou uma progressão anual de 7\%, com 16.291 casos (AZIZA YDA, et al., 2019). No Brasil, de 2016 a 2017, foi estimado em 12.600 novos casos nessa faixa etária (ABDOLJABBARI M, et al., 2018; MEDEIROS EGMS, et al., 2019). Devido à alta taxa de mortalidade, as neoplasias pediátricas são consideradas um problema de saúde pública, principalmente nos países em desenvolvimento, onde as taxas de sobrevivência são inferiores a $35 \%$, enquanto os países desenvolvidos atingem 85\% (AZIZA YDA, et al., 2019; MOLINARO ML e FLETCHER PC, 2018).

O diagnóstico de CJ é devastador e estressante para toda a família, gerando sofrimento e desestruturação, pois as crianças representam o futuro e a esperança, os pais temem o futuro de suas vidas, e o ser cuidador pode se tornar uma situação desafiadora. Com a possibilidade cada vez mais frequente de tratamento oncológico ambulatorialmente, os cuidadores passam em media 32,9 horas por semana em tarefas de assistência e 72\% realizam tarefas médicas ou de enfermagem (VITORINO LM, et al., 2018).

Nessa perspectiva, ser o cuidador pode se tornar uma situação desafiadora, com acometimento frequente de ansiedade e depressão que podem perdurar além das fases de tratamento e remissão, particularmente naqueles cuidadores cujas crianças têm prognóstico ruim ou não sobrevivem à doença (MEDEIROS EGMS, et al., 2019; VITORINO LM, et al., 2018; TANCO K, et al., 2017).

Até um quarto dos cuidadores de câncer podem apresentar sintomas depressivos graves, e um quinto a um terço da ansiedade intensa. Esse sofrimento pode ser pior no contexto do câncer em estágio avançado. Embora seja problemático por si só, o estresse emocional também está associado à pior qualidade de vida, maior carga percebida no cuidado, distúrbios do sono e disfunções do sistema imunológico. Em alguns estudos, níveis mais altos de sintomas nos cuidadores estão associados à um maior sofrimento do paciente, sugerindo que o sofrimento do cuidador pode impactar negativamente também no paciente (TANCO K, et al., 2017).

Para avaliar a extensão do dano sofrido pelos cuidadores, o presente estudo teve como objetivo avaliar características sociodemográficas e os sintomas de ansiedade/depressão em cuidadores de crianças e adolescentes diagnosticados com neoplasias malignas.

\section{MÉTODOS}

Trata-se de um estudo transversal, exploratório, com abordagem quantitativa. Participaram da pesquisa 45 cuidadores, com acompanhamento regular de crianças de 0 a 19 anos, em tratamento oncológico em duas unidades de referência localizadas em uma capital do nordeste brasileiro. Os dados coletados foram entre os dias 06 e 30 de abril de 2019.

Os cuidadores que compuseram a amostra foram convidados a participar da pesquisa no período em que permaneceram nos setores de tratamento. Após a aceitação, foram informados sobre os objetivos do estudo e assinaram o Termo de Consentimento Livre e Esclarecido (TCLE).

A população foi constituída por cuidadores de crianças e adolescentes em tratamento para neoplasias malignas, tendo em parâmetros os níveis de sobrecarga emocional enfrentados pelos entrevistados. $O$ questionário aplicado aos cuidadores, foi originalmente criado na Alemanha em 1993, traduzido para o inglês em 1995 e validado no Brasil por Medeiros et al, em 1998. 
Foram incluídos cuidadores regular de crianças com faixa etária compreendida entre 0 a 19 anos, com neoplasias malignas, em tratamento clínico e cirúrgico, radioterapia e/ou quimioterapia, em uma capital do nordeste brasileiro. Empregou-se como critério de exclusão a criança que estava sendo tratada de uma neoplasia benigna; estava em tratamento há menos de dois meses e o cuidador atuava ocasionalmente no tratamento de crianças com neoplasias malignas.

Foi aplicado o inventário de ansiedade/depressão de Beck (BDI) para avaliar presença e grau dos sinais de ansiedade/depressão dos cuidadores. O Inventário de Ansiedade de Beck (BAI) consiste em 21 questões sobre como o indivíduo tem se sentido na última semana, expressas em sintomas comuns de ansiedade (como sudorese e sentimentos de angústia). Cada pergunta tem quatro respostas possíveis, e a que mais se parece com o estado mental do indivíduo deve ser sinalizada. As respostas possíveis são: não; um pouco: não me incomodou muito; moderadamente: era desagradável, mas eu aguentava; severamente: o BAl quase sem suporte pode ter uma pontuação máxima de 63 e as categorias são: 0-10: grau mínimo de ansiedade; 11-19: ansiedade leve; 20-30 ansiedade moderada e 31-63 ansiedade grave.

O Inventário de Depressão de Beck consiste em 21 itens, que incluem sintomas e atitudes. As respostas variam de 0 (sem sintomas) a 3 (sintomas depressivos mais graves). Os itens referem-se a tristeza, pessimismo, sentimento de fracasso, falta de satisfação, sentimento de culpa, sentimento de punição, autodepreciação, autoacusação, idéias suicidas, crises de choro, irritabilidade, retraimento social, indecisão, distorção da imagem corporal, inibição para o trabalho, distúrbios do sono, fadiga, perda de apetite, perda de peso, preocupação somática, diminuição da libido. Existem várias propostas de corte para distinguir os níveis de depressão, mas para amostras não diagnósticas, recomenda-se pontuação acima de 15 para disforia e acima de 20 para depressão. Em sua versão atual, o questionário foi desenvolvido para pacientes com mais de 13 anos de idade e é amplamente utilizado como ferramenta de medição por profissionais de saúde e pesquisadores em diversos contextos clínicos e de pesquisa.

Pontuações variando de 0 (zero) a 3 (três) pontos são dadas para avaliar o grau de carga do cuidador em cada um dos 28 itens respondidos. O valor total obtido com a soma desses pontos se ajusta ao avaliado dos três graus da escala. $O$ grau I corresponde a uma pontuação entre 0 (zero) e 41 (quarenta e um) pontos e traduz que o cuidador apresenta baixo nível de estresse e não corre risco de sintomas físicos e psicossomáticos; O grau II corresponde a uma pontuação entre 42 (quarenta e dois) e 55 (cinquenta e cinco) pontos, revelando que o cuidador apresenta um nível moderado de estresse e um risco considerável de sintomas físicos e psicossomáticos; Finalmente, o Grau III corresponde a uma pontuação entre 56 (cinquenta e seis) e 84 (oitenta e quatro) pontos e revela que o cuidador está sendo submetido a um nível de estresse alto a muito alto e tem um risco muito alto de sofrer de problemas físicos. e sintomas psicossomáticos (MEDEIROS EGMS, et al., 2019).

Os dados foram analisados e interpretados por estatística descritiva e inferencial. As variáveis categóricas foram apresentadas usando frequências simples e relativas variáveis numéricas mostradas como média e desvio padrão. Na parte inferencial, foi utilizado o teste de correlação de Pearson, entre a soma dos escores das escalas de Ansiedade e Depressão de Beck e CaregiverBurden. O teste do qui-quadrado também foi utilizado para encontrar possíveis associações entre as variáveis que representam o grau de ansiedade, depressão e sobrecarga do cuidador. O nível estipulado de significância estatística foi estabelecido em $5 \%$ ( $p$ $\leq 0,05)$ e todos os testes foram bicaudais. O software selecionado para as análises foi o Statistical Package for the Social Sciences (IBM SPSS 25.0).

Este estudo foi submetido ao Comitê de Ética em Pesquisa (CEP) da Universidade Tiradentes (UNIT), em conformidade com a Resolução 466/12 do Conselho Nacional de Saúde, tendo sido aprovado pelo parecer no 3.239.027 de 02/02/2019.

\section{RESULTADOS}

Participaram do estudo 45 cuidadores, com idade média de 34,4 ( $\pm 10,08$ anos), sendo predominantemente do sexo feminino $(91,1 \%)$, com relacionamento materno $(84,4 \%)$, ensino fundamental $(48,9 \%)$ e com renda familiar inferior a um salário-mínimo $(51,1 \%)$ (Tabela 1). 
Tabela 1 - Característicassociodemográficas de cuidadores de crianças e adolescentes com neoplasias malignas em tratamento.

\begin{tabular}{lc}
\hline Características & Frequência (45) \\
\hline Idade cuidador & $34,42 \pm 10,08$ \\
\hline Gênero & $4(8,9)$ \\
\hline Masculino & $41(91,1)$ \\
Feminino & \\
\hline Escolaridade & $3(6,37)$ \\
\hline Ausente & $22(48,9)$ \\
Fundamental & $16(35,6)$ \\
Médio & $4(8,9)$ \\
Superior & \\
\hline Renda familiar & $23(51,1)$ \\
\hline < 1 salário mínimo & $21(46,7)$ \\
1 a 2 salários mínimo & $1(2,2)$ \\
3 a 4 salários mínimo & \\
\hline Parentesco & $38(84,4)$ \\
\hline Mãe & $2(4,4)$ \\
Pai & $2(4,4)$ \\
Avó & $1(2,2)$ \\
Tio & $1(2,2)$ \\
Tia & $1(2,2)$ \\
Irmão &
\end{tabular}

Fonte: Carvalho WMO, et al., 2020.

Os pacientes com câncer eram em sua maioria meninos $(66,7 \%)$ na faixa etária de 1 e 4 anos $(44,4)$. 0 câncer mais frequente foi a leucemia (51,1\%), o ano com maior número de casos foi 2018 (44,6\%), o tratamento mais frequente foi a quimioterapia (91,1\%). O perfil clínico-epidemiológico encontra-se na (Tabela 2).

Tabela 2 - Perfil epidemiológico de crianças e adolescentes com neoplasias malignas em tratamento.

\begin{tabular}{lc}
\hline Características & Frequência (45) \\
\hline Idade média & $7,31( \pm 5,45)$ \\
\hline Sexo & \\
\hline Menino & $30(66,7)$ \\
Menina & $15(33,3)$ \\
\hline Procedência & $15(33,3)$ \\
\hline Aracaju & $3(6,7)$ \\
Grande Aracaju & $18(40,0)$ \\
Interior & $9(20,0)$ \\
Outro Estado & \\
\hline Neoplasia & $23(51,1)$ \\
\hline II & $1(2,2)$ \\
III & $4(8,9)$ \\
IV & $3(6,7)$ \\
VI & $4(8,9)$ \\
VII & $2(4,4)$ \\
VIII & $1(2,2)$ \\
IX & $2(4,4)$ \\
XI & $2(4,4)$ \\
XII & $3(6,7)$ \\
\hline Ano de diagnóstico & \\
\hline 2006 & $1(2,2)$ \\
2007 & $1(2,2)$ \\
2012 & $1(2,2)$ \\
2013 & $1(2,2)$ \\
2014 & $2(4,4)$ \\
2016 & $4(8,9)$ \\
2017 & $5(11,1)$ \\
2018 & $20(44,4)$ \\
2019 & $10(22,2)$ \\
\hline Tratamento & \\
\hline Cirúrgico & $15(33,3)$ \\
Quimioterapia & $41(91,1)$ \\
Radioterapia & $11(24,4)$ \\
Clínico & $15(33,3)$ \\
\hline
\end{tabular}

Fonte: Carvalho WMO, et al., 2020. 
A maioria dos cuidadores apresentava sintomas graves de ansiedade $(77,8 \%)$ e os sinais de depressão foram na maioria mínimos $(37,8 \%)$ ou leves $(35,6 \%)$. Os sintomas de burnout não foram predominantes na amostra, com a maioria dos cuidadores apresentando Nível de Estresse Grau I (62,2\%) com baixo risco de sintomas físicos e psicológicos (Tabela 3).

Tabela 3 - Níveis de ansiedade, depressão e sobrecarga em cuidadores de crianças e adolescentes em tratamento para neoplasia maligna.

\begin{tabular}{lcc}
\hline Características & Frequência (45) & Min - Max \\
\hline Ansiedade Beck & $40,78 \pm 12,87$ & $7-63$ \\
\hline Mínima & $1(2,2)$ & \\
Leve & $0(0,0)$ & \\
Moderada & $9(20,0)$ & \\
Grave & $35(77,8)$ & $0-50$ \\
\hline Depressão Beck & $17,18 \pm 12,62$ & \\
\hline Mínima & $17(37,8)$ & \\
Leve & $16(35,6)$ & \\
Moderada & $5(11,1)$ & \\
Grave & $7(15,6)$ & \\
\hline Sobrecargacuidador & $36,96 \pm 15,83$ & \\
\hline Grau I & $28(62,2)$ & \\
Grau II & $11(24,4)$ & \\
Grau III & $6(13,3)$ & \\
\hline
\end{tabular}

Fonte: Carvalho WMO, et al., 2020.

Não houve associação entre os níveis de ansiedade e a escala de sobrecarga do cuidador $(p=0,163)$. Em todos os níveis de carga, há um número baixo de pacientes com ansiedade mínima e um número alto de pacientes com ansiedade severa. Por outro lado, há uma correlação entre depressão e sobrecarga do cuidador $(p<0,001)$ (Tabela 4$)$.

Tabela 4 - Correlação entre a carga do cuidador com os níveis de ansiedade e depressão.

\begin{tabular}{|c|c|c|c|c|}
\hline \multirow{2}{*}{ Características } & \multicolumn{3}{|c|}{ Sobrecarga } & \multirow[b]{2}{*}{$\mathbf{p}$} \\
\hline & Grau I & Grau II & Grau III & \\
\hline Ansiedade & & & & 0,163 \\
\hline Mínima & $1(3,6)$ & $0(0,0)$ & $0(0,0)$ & \\
\hline Leve & $0(0,0)$ & $0(0,0)$ & $0(0,0)$ & \\
\hline Moderada & $7(25,0)$ & $2(18,2)$ & $0(0,0)$ & \\
\hline Grave & $20(71,4)$ & $9(81,8)$ & $6(100,0$ & \\
\hline Depressão & & & & $<0,001^{*}$ \\
\hline Mínima & $15(53,6)$ & $2(18,2$ & $0(0,0)$ & \\
\hline Leve & $9(32,1)$ & $6(54,5)$ & $1(16,7)$ & \\
\hline Moderada & $1(3,6)$ & $3(27,3)$ & $1(16,7)$ & \\
\hline Grave & $3(10,7)$ & $0(0,0)$ & $4(66,7)$ & \\
\hline
\end{tabular}

Legenda: valor significante $(p \leq 0,05)$.

Fonte: Carvalho WMO, et al., 2020.

Foi observado uma associação entre os níveis de ansiedade e depressão $(p=0,005)$ e o aumento da ansiedade foi associado a níveis mais altos de depressão, como visto na (Tabela 5).

Tabela 5 - Correlação dos níveis de ansiedade e depressão em cuidadores de crianças e adolescentes tratados por neoplasias malignas.

\begin{tabular}{|c|c|c|c|c|c|}
\hline \multirow{2}{*}{ Características } & \multicolumn{4}{|c|}{ Depressão } & \multirow[b]{2}{*}{$p$} \\
\hline & Mínima & Leve & Moderada & Grave & \\
\hline Ansiedade & & & & & $0,005^{\star}$ \\
\hline Mínima & $1(5,9)$ & $0(0,0)$ & $0(0,0)$ & $0(0,0)$ & \\
\hline Leve & $0(0,0)$ & $0(0,0)$ & $0(0,0)$ & $0(0,0)$ & \\
\hline Moderada & $7(41,2)$ & $2(12,5)$ & $0(0,0)$ & $0(0,0)$ & \\
\hline Grave & $9(52,9)$ & $14(87,5)$ & $5(100,0)$ & $7(100,0)$ & \\
\hline
\end{tabular}

Legenda: valor significante $(p \leq 0,05)$.

Fonte: Carvalho WMO, et al., 2020. 
A idade e a ansiedade e depressão do cuidador não mostraram uma associação forte. Os níveis de ansiedade e depressão foram semelhantes em cuidadores com renda mais baixa e a mais alta, e os níveis mais baixo e mais alto (Tabela 6 ).

Tabela 6 - Correlação entre ansiedade, depressão e idade do cuidador.

\begin{tabular}{cc}
\hline Correlação & Idade do cuidador \\
\hline Ansiedade & $-0.204(\mathrm{~ns})$ \\
Depressão & $0.065(\mathrm{~ns})$
\end{tabular}

Fonte: Carvalho WMO, et al., 2020.

\section{DISCUSSÃO}

No presente estudo, os cuidadores tinham idade média de 34 anos, semelhante aos resultados encontrados por Beck ARM e Lopes MHBM (2007), em um estudo descritivo, comparativo e transversal com 50 cuidadores, em Campinas (SP), cujo a idade média foi de 33,6 anos. Gelesson DD, et al. (2009), em estudo descritivo de natureza mista, realizado no Ambulatório de Quimioterapia do Instituto de Oncologia Pediátrica (IOP-GRAACC), em São Paulo (SP), encontraram uma média de 33,4 anos, e Benchaya I, et al. (2014), ao descrever os efeitos da educação e treinamento dos pais nos comportamentos de cuidadores de crianças diagnosticadas com câncer observaram média de 30 anos de idade.

A predominância feminina encontrada neste estudo pode ser atribuída ao cuidado materno, como discutido em vários estudos (BECK ARM e LOPES MHBM, 2007; NAM GE, et al., 2016). O gênero feminino também foi descrito como predominante por Demirtepe-Saygili D e Bozo O (2011) quando examinaram o papel moderador e o apoio social percebidos como indicadores de bem-estar entre cuidadores de crianças com leucemia. Os mesmos achados são corroborados por Paula DPS, et al. (2019), em um estudo descritivo, transversal e qualitativo, realizado no Centro de Alta Complexidade Oncológica Irmã Malvina de Montes Claros (MG), em 27 cuidadores familiares de crianças e adolescentes.

Em relação à escolaridade, a maioria dos cuidadores possuía apenas o ensino fundamental. Da mesma forma, o baixo nível de escolaridade foi verificado nos estudos desenvolvidos por Santo EARE, et al. (2011), em um estudo transversal com 32 cuidadores durante o tratamento quimioterápico, o qual se correlaciona no estudo com o aumento da depressão. Por outro lado, Beck ARM e Lopes MHBM (2007) e Benchaya I, et al. (2014) relataram em seus estudos a predominância do ensino fundamental incompleto. O baixo perfil educacional estava intimamente relacionado à baixa renda familiar, que no presente estudo a maioria dos pacientes possuía uma renda mensal inferior a um salário mínimo. Achado semelhante foi encontrado em estudo brasileiro, o que destaca o ônus do baixo desenvolvimento social e econômico no Brasil como um fator importante que afeta os níveis de depressão e ansiedade entre os cuidadores (PAULA DPS, et al., 2019).

Além disso, a média de idade das crianças do presente estudo foi de 7,31 anos, acima da descrita por Beck ARM e Lopes MHBM (2007) que exibiram 5,9 anos e por Gelesson DD et al. (2009) com 5,2 anos. Pedrosa A, et al. (2015), ao estudar o perfil clínico-epidemiológico de pacientes pediátricos com câncer em um hospital de referência do Piauí (Brasil), encontrou que as crianças em tratamento de câncer tinham idade de 1 a 4 anos, o que apresenta uma amostra heterogênea quando comparada a, e Silva Junior, PF et al. (2016), em pesquisa desenvolvida na instituição que acolhe crianças com câncer, em Criciúma (SC), que estudou cuidadores de crianças de 8 a 9 anos (SILVA JUNIOR PF, et al., 2015 ; PEDROSA A, et al., 2015). Não foram encontradas correlações com idade e depressão ou ansiedade nos estudos atuais e anteriores.

Os achados deste estudo constataram uma predominância do câncer em crianças do sexo masculino (66,7\%). Hintz LG, et al. (2019) e Monteiro NM, et al. (2018), do mesmo modo, encontraram que a maioria dos casos de crianças com câncer pertenciam ao sexo masculino, com 53,3\% e 55,0\%, respectivamente. Diniz AG, et al. (2005) relataram que o câncer se apresenta com maior incidência no sexo masculino, podendo indicar uma fragilidade constitucional no gênero masculino ou uma associação com fatores genéticos de predisposição ligadas ao cromossomo X. 
Apesar de o percentual de origem das crianças em tratamento de neoplasias malignas ser maior do interior do Estado, os valores são muito próximos aos da capital, que apresenta maior prevalência por ser uma área densamente povoada, com $25 \%$ da população do Estado (IBGE, 2018).

A malignidade mais frequente que afetou as crianças em tratamento foi a leucemia, resultado do estudo ao descrever os diagnósticos e os cuidados de enfermagem prestados aos pacientes com câncer de infância e juventude, em um estudo retrospectivo, com abordagem quantitativa, realizado a partir de prontuários no arquivo médico. O departamento de um CACON, em crianças e adolescentes internados na clínica pediátrica, no período de 2011 e 2012, também mostrou leucemia como a neoplasia mais prevalente (BECK ARM e LOPES MHBM, 2007; GELESSON DD, et al., 2009; PAULA DPS, et al., 2019; JUNIOR PFS, et al., 2015). Estudos mundiais corroboram com esses achados, onde crianças de 0 a 19 anos correspondem a cerca de 25,6\% dos casos de câncer (CHATENOUD L, et al., 2010; STELIAROVA-FOUCHER E, et al., 2017).

O tipo mais comum de tratamento utilizado foi a quimioterapia. Benchaya I, et al. (2014) relataram que, independentemente do diagnóstico, todas as crianças foram submetidas à quimioterapia em regime ambulatorial.

O tratamento oncológico é doloroso fisicamente e emocionalmente tanto para a criança como para a família, a retirada do seu convívio familiar e do seu lar para uma instituição, que muitas vezes, fica longe é extremamente sacrificante. Brum MV e Aquino GB (2014) apontam que as famílias oscilam entre otismismo e incertezas frente a todo processo do tratamento oncológico, sendo importante visar as condições biopsicossociais que rodeiam a criança, para que ela e toda sua família recebam apoio e atenção integral.

Neste estudo, a maioria dos cuidadores apresentaram sinais e sintomas de ansiedade classificados como graves e sintomas depressivos classificados como mínimos e leves. Alto nível de ansiedade dos pais durante o tratamento do câncer infantil foi evidenciado por Brum MV e Aquino GB (2014) em pesquisa realizada na Fundação Cristiano Varella, Hospital do Câncer, no município de Muriaé (MG), aplicada a 10 cuidadores de crianças em tratamento oncológico, inventário e escala de Beck, resultando em um total de $80 \%$. Segundo Patiño-Fernandez AM, et al. (2008), ao realizar pesquisas em que estabeleceram que o Transtorno de Estresse Agudo (TEA) e os Sintomas Subclínicos de Estresse Agudo (SAS) podem ser uma estrutura útil para entender as reações psicológicas de mães e pais dos recém-nascidos diagnosticados com malignidade pediátrica, as pessoas com comportamentos ansiosos podem ter situações difíceis de gerenciar, que, no contexto adverso do câncer, podem afetar consideravelmente a qualidade do atendimento ao paciente.

Níveis de depressão também são identificados em cuidadores de crianças em tratamento de câncer. Neste estudo, os níveis de depressão também foram significativos, correspondendo a 26,7\%. Segundo Santo EARE, et al. (2011), a presença da criança na família está ligada a sentimentos positivos e a descoberta do câncer gera medo e desesperança, predispondo à depressão. Níveis clinicamente relevantes de sintomas depressivos foram registrados: $25 \%$ observado por Aziza YDA, et al. (2019), em um estudo transversal que teve como objetivo investigar as necessidades e fatores associados ao sofrimento psicológico dos pais ao cuidar de crianças com câncer em pediatria enfermarias de dois hospitais na Indonésia, 28\% por Warmerdam JV, et al. (2019) ao realizar uma meta-análise para determinar a prevalência de doença mental em pais de crianças com câncer e 35\% por Rahmani A, et al. (2018) em um estudo descritivo-correlacional ao avaliar ansiedade e depressão em 148 pais de crianças com câncer internadas em um hospital pediátrico afiliado à Universidade de Ciências Médicas em Tabriz, Irã.

O ônus para os cuidadores de crianças com doenças crônicas está diretamente associado ao grau de predisposição para o desenvolvimento de sintomas depressivos e ansiosos. A maior parte do nível de sobrecarga encontrada neste estudo foi de um grau mínimo, o que determina um baixo risco de desenvolver sintomas depressivos e ansiosos. Ao comparar o grau de sobrecarga com os níveis de ansiedade e depressão, observou-se uma relação crescente entre depressão e sobrecarga. Em relação à ansiedade, o mesmo comportamento não foi observado, pois havia níveis significativamente elevados de ansiedade, independentemente do grau de sobrecarga do cuidador. 
Existem limitações nos vários estudos desenvolvidos sobre esse tema. O principal envolve a falta de uniformidade nas ferramentas para avaliar os níveis de ansiedade e depressão. Isso se deve à diversidade de instrumentos disponíveis para avaliar o mesmo contexto. Portanto, de acordo com Warmerdam JV, et al. (2019), a padronização das escalas e pontos de corte usados para descrever a presença de ansiedade, depressão e outros distúrbios, permitiria comparações mais precisas entre os estudos. Além disso, a maioria dos estudos, como este, envolve pesquisas transversais, as quais, como afirma Klassen A, et al. (2007), ao descrever uma revisão de literatura realizada para identificar fatores importantes que foram investigados como explicações da variabilidade na saúde e no bem-estar dos pais de crianças com câncer, eles não conseguem avaliar os processos de enfrentamento e adaptação ao longo de sua experiência com o câncer. É necessário superar essas dificuldades e criar um processo de avaliação e apoio para essas famílias, com o objetivo de otimizar os cuidados e promover bem-estar psicológico para pais e pacientes.

\section{CONCLUSÃO}

A idade média do cuidador na amostra foi de 34 anos, predominantemente do sexo feminino, com relacionamento materno, ensino fundamental e renda familiar abaixo de um salário-mínimo. A maioria dos cuidadores apresentava sinais e sintomas graves de ansiedade e estava minimamente ou levemente deprimida. Os cuidadores foram classificados como apresentando baixo nível de estresse e menor risco de sintomas físicos e psicossomáticos. Não houve associação entre sobrecarga do cuidador e níveis de ansiedade. No entanto, níveis mais altos de sobrecarga do cuidador correlacionaram-se com maiores escores de depressão. Finalmente, o aumento da ansiedade foi associado a sintomas depressivos.

\section{AGRADECIMENTOS}

Em memória a Walter Marcelo, agradecemos por sua contribuição à comunidade acadêmica enquanto pesquisador, professor e médico que atuou em prol à saúde pública do estado de Sergipe. Certamente as suas contribuições foram sementes plantadas com muito sacrifício e zelo, outrora serão colhidos frutos doces por àqueles que herdaram seus ensinamentos

\section{REFERÊNCIAS}

1. ABDOLJABBARI M, et al. Taking Refuge in Spirituality, a Main Strategy of Parents of Children with Cancer: a Qualitative Study. Asian Pac J Cancer Prev APJCP, 2018; 19(9): 2575-2580.

2. AZIZA YDA, et al. Unmet supportive care needs and psychological distress among parents of children with cancer in Indonesia. Psychooncology, 2019; 28(1): 92-98.

3. BECK ARM, LOPES MHBM. Cuidadores de crianças com câncer: aspectos da vidaafetadospelaatividade de cuidador. Rev Bras Enferm, 2007; 60(6): 670-675.

4. BENCHAYA I, et al. Efeitos de instrução e de treino parental emcuidadores de crianças com câncer. PsicolTeor E Pesqui, 2014; 30(1): 13-23.

5. BRUM MV, AQUINO GB. Estudo do impacto do tratamento do câncerinfantil nos aspectosemocionais dos cuidadores de crianças com diagnóstico da doença. Revista Científica Da Faminas, 2014;10(2):21.

6. CHATENOUD L, et al. Childhood cancer mortality in America, Asia, and Oceania, 1970 through 2007. Cancer, 2010; 116(21): 5063-5074.

7. DEMIRTEPE-SAYGILI D, BOZO Ö. Perceived social support as a moderatoroftherelationshipbetweencaregiverwellbeingindicatorsandpsychologicalsymptoms. Journalof Health Psychology, 2011; 16(7): 1091-1100.

8. DINIZ AB, et al. Perfil epidemiológico do câncer infantil em população atendida por uma unidade de oncologia pediátrica em Salvador-Bahia. Revista de Ciências Médicas e Biológicas, 2005; 4(2): 131-139.

9. GELESSON DD, et al. Significado da neutropenia e necessidades de cuidadoemdomicílio para oscuidadores de crianças com câncer. Rev Lat Am Enfermagem, 2009; 17(6): 933-939.

10. HINTZ LG, et al. Perfil clínico-epidemiológico de crianças e adolescentes em tratamento oncológico. Ciência \& Saúde, 2019; 12(1): e31421.

11. IBGE. Institutobrasileiro de geografia e estatística. IndicadoresSociais: umaanálise dos resultados do universo do CensoDemográfico 2018. Rio de Janeiro: IBGE, 2018.

12. JUNIOR PFS, et al. O perfil dos pacientesatendidosna casa guido e a importância do uso do brinquedoterapêuticocomo forma de cuidadoaopacientepediátrico para profissionaisqueatendemcrianças com câncer. Rev Interdiscip Estud Em Saúde, 2015; 4(2): 63-78.

13. KARLSON CW, et al. Development of the family symptom inventory: a psychosocial screener for children with hematology/oncology conditions. J Pediatr Hematol Oncol, 2015;37(2):140-146. 
14. KLASSEN A, et al. Developing a literature base to understand the caregiving experience of parents of children with cancer: a systematic review of factors related to parental health and well-being. Support Care Cancer Off $\mathrm{J}$ Multinatl Assoc, 2007; 15(7): 807-818.

15. MEDEIROS EGMS, et al. Repercussões do câncerinfantil no cotidianodo familiar cuidador. Rev Rene, 2014;15(2).

16. MOLINARO ML, FLETCHER PC. The Balancing Act: Mothers' Experiences of Providing Care to Their Children With Cancer. Journal of Pediatric Oncology Nursing, 2018; 35(6): 439-446.

17. MONTEIRO NML, et al. Perfil Clínico e Epidemiológico dos Pacientes de um Serviço de Oncologia Pediátrica de um Hospital do Leste de Minas Gerais. Rev Med Minas Gerais, 2018; 28: 1-5.

18. NAM GE, et al. Understanding psychological distress among pediatric cancer caregivers. Supportive Care in Cancer, 2016; 24(7): 3147-3155.

19. PALACIO C, et al. The influence of psychological factors on the burden of caregivers of patients with advanced cancer: Resiliency and caregiver burden. Palliat Support Care, 2018; 16(3): 269-277.

20. PATIÑO-FERNÁNDEZ AM, et al. Acute stress in parents of children newly diagnosed with cancer. Pediatr Blood Cancer, 2008; 50(2): 289-292.

21. PAULA DPS, et al. Câncer infanto juvenil do âmbito familiar: percepções e experiências frente ao diagnóstico. Rev Cuid, 2019; 10(1).

22. PEDROSA AO, et al. Perfil clínico-epidemiológico de clientes pediátricos oncológicos atendidos em um hospital de referência do Piauí. Rev Interdiscip, 2015; 8(3): 12-21.

23. RAHMANI A, et al. Anxiety and Depression: A Cross-sectional Survey among Parents of Children with Cancer. Indian J Palliat Care, 2018;24(1):82-85.

24. SANTANA ME, et al. O cuidar em oncologia pediátrica: um estudo baseado no processo de enfermagem. Rev Destaques Acadêmicos, 2017; 9(3).

25. SANTO EARE, et al. Taking care of children with cancer: evaluation of the caregivers' burden and quality of life. Rev Lat Am Enfermagem, 2011; 19(3): 515-522.

26. SILVA JUNIOR PF, et al. O perfil dos pacientes atendidos na casa guido e a importância do uso do brinquedo terapêutico como forma de cuidado ao paciente pediátrico para profissionais que atendem crianças com câncer. Revista Interdisciplinar de Estudos em Saúde, 2015; 4(2): 63-78.

27. STELIAROVA-FOUCHER E, et al. International incidence of childhood cancer, 2001-10: a population-based registry study. Lancet Oncol, 2017; 18(6): 719-731.

28. TANCO K, et al. A systematic review of instruments assessing dimensions of distress among caregivers of adult and pediatric cancer patients. Palliat Support Care, 2017; 15(1): 110-124.

29. VITORINO LM, et al. Spiritual and religious coping and depression among family caregivers of pediatric cancer patients in Latin America. Psychooncology. 2018; 27(8): 1900-1907.

30. WARMERDAM JV, et al. Prevalence of anxiety, depression, and posttraumatic stress disorder in parents of children with cancer: A meta-analysis. Pediatr Blood Cancer, 2019; 66(6): e27677. 